\title{
Fungal Coronary Emboli Presenting as ST-Segment Elevation Myocardial Infarction
}

\author{
Khola S Tahir ${ }^{1}$, Akinniran Abisogun ${ }^{1}$, David Lee ${ }^{1}$ and Xuming Dai ${ }^{1,2 *}$ \\ ${ }^{1}$ Division of Cardiology, University of North Carolina at Chapel Hill, USA \\ ${ }^{2}$ McAllister Heart Institute, University of North Carolina at Chapel Hill, USA
}

Submission: June 22, 2017; Published: July 05, 2017

*Corresponding author: Xuming Dai, Division of Cardiology, University of North Carolina at Chapel Hill,160 Dental Circle, CB \#7075, Chapel Hill, NC 27599, Tel: 984-974-5956; Fax: 984-974-4366; Email:xuming_dai@med.unc.edu

\begin{abstract}
Coronary embolization is an uncommon cause of ST elevation myocardial infarction (STEMI). Fungal endocarditis associated coronary embolization presented as STEMI is very rare. We here reported a bioprosthetic aortic valve associated fungal endocarditis presented as a STEMI resulted from fungal coronary embolization. Aspirational thrombectomy and balloon angioplasty was unsuccessful in reperfusion therapy. We discussed the challenges in primary percutaneous coronary intervention and unfavorable prognosis of this entity.
\end{abstract}

Keywords: Septic emboli; STEMI; Fungal endocarditis

\section{Introduction}

Fungal endocarditis is a rare infection but occurs more commonly in patients with bioprosthetic valves than native valves. Systemic embolization is a well-known complication of fungal endocarditis. Coronary embolization is rare but when it does occur, carries a high mortality rate. Here we present the case of a patient with Candida albicans endocarditis who had an acute occlusion of an epicardial coronary artery by fungal emboli confirmed by culture of aspirational thrombectomy products.

\section{Case Presentation}

A 22-year-old female with a history of intravenous drug use and aortic valve bacterial endocarditis, for which she required aortic valve replacement with a bioprosthetic valve. The patient presented to an outside hospital with worsening fatigue. Blood culture confirmed Candida Albicans fungemia. Transthoracic echocardiography (TTE) showedaleftventricularejectionfraction (LVEF) of $45 \%$ and a well-seated bioprosthetic aortic valve with moderately limited excursion, mild aortic regurgitation, and a $0.8 \mathrm{~cm}$ mass on one of the prosthetic valve leaflets consistent with large vegetation on the bioprosthetic aortic valve in the setting of fungemia. Broad spectrum intravenous anti-fungal therapy was initiated. Subsequently, radiographic evidence of mycotic aneurysms involving the celiac and superior mesenteric arteries as well as splenic infarcts was found. These mycotic aneurysms underwent coiling therapy by interventional radiology. The patient made promising progress and had stabilized clinically in the early phase of hospitalization.

On hospitalization day 13, the patient suffered a pulseless electrical activity (PEA) arrest and was successfully resuscitated. Post-resuscitation 12-lead electrocardiogram (ECG) revealed inferior ST-segment elevations with reciprocal anterolateral ST-segment depressions (Figure 1A). Emergent bedside TTE revealed inferior and inferoseptal wall akinesis with estimated global LVEF of 10-15\%. Emergent coronary angiography found that there was acute total occlusion in the mid segment of the large, dominant left circumflex artery with TIMI 0 flow (Figure 1B) considered as the culprit lesion for the STEMI No significant atherosclerotic disease was apparent in other major epicardial coronary arteries. After wiring the lesion, aspirational thrombectomy using Priority One ${ }^{\circledR}$ aspirational catheter (Terumo Inventional Systems) was performed in the consideration of large thrombus burden without underlying atherosclerotic stenosis. Large amount of yellowish debris with minimal blood stain was retrieved after multiple passes (Figure 1C). Subsequent balloon angioplasty was also performed. No-reflow phenomenon persisted despite repeated efforts of 
aspiration, balloon angioplasty and intra-coronary adenosine administrations. Large burden of distal fungal emboli was thought to be the cause of no-reflow. Cultures of the aspiration contents from the circumflex artery were subsequently positive for Candida albicans, which was identical to previous blood culture growth. Non-selective aortic root angiography revealed evidence of aortic paravalvular abscess with communications between the aorta and pulmonary artery. Unsuccessful revascularization for embolic occlusion of large dominant circumflex led to pump failure and cardiogenic shock, as well as worsening of septic shock led to rapid clinical deterioration requiring increasing vasopressor support. Emergent surgical intervention was ultimately deferred citing prohibitively high risk for re-do aortic valve replacement in the setting of fungal endocarditis, paravalvular abscess, acute MI and mixed shock, to which the patient succumbed three days later.

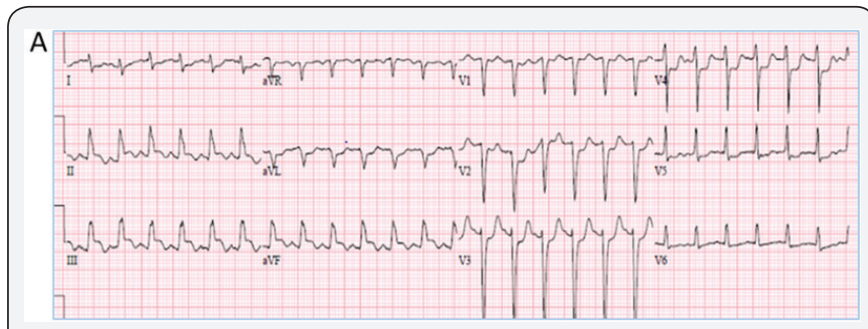

Figure 1A: STEMI ECG: ECG after resuscitation revealed sinus tachycardia, ST elevations in II, III, avF and ST depressions in V2-V5, I, aVL was diagnostic for inferoposterior STEMI.

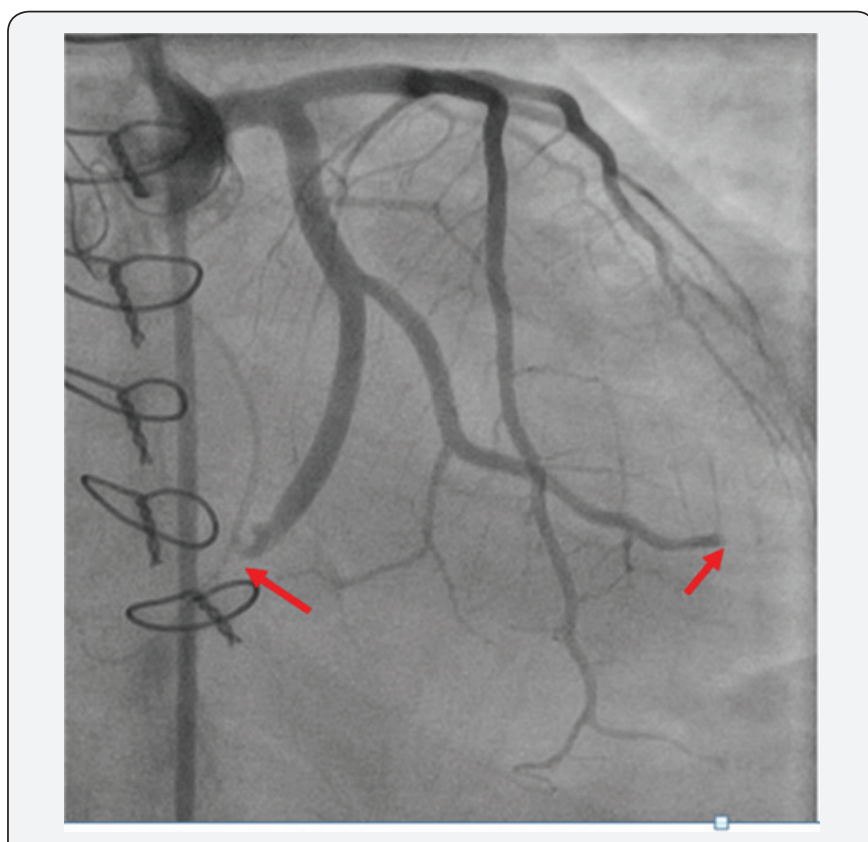

Figure 1B: Coronary angiography: Emergent coronary angiography revealed acute thrombotic occlusion of dominant circumflex and obtuse marginal branch (red arrows). There is no evidence of underlying atherosclerotic disease in other areas of epicardial coronary arteries.

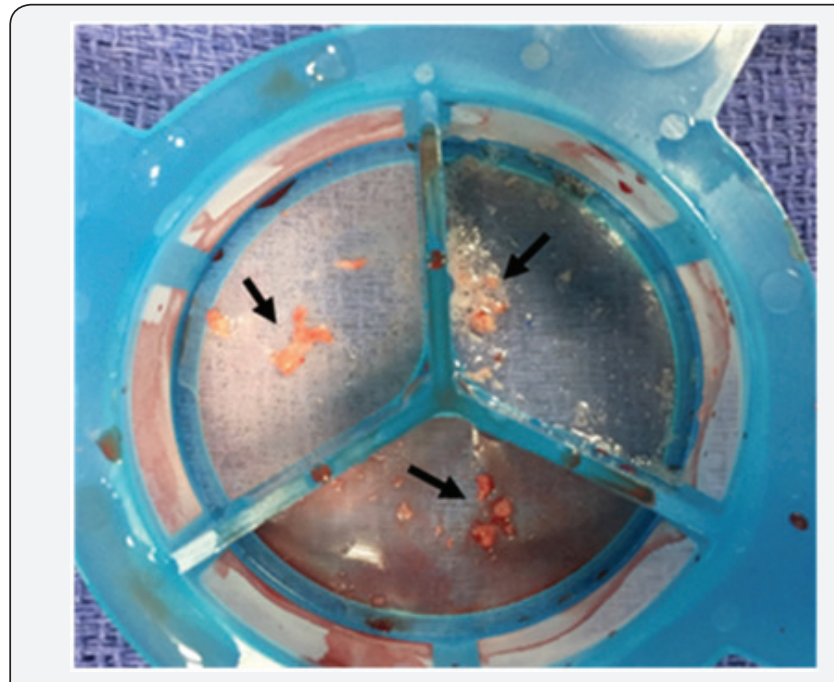

Figure 1C: Aspirational products from thrombectomy of the occluded circumflex: large amount of yellowish debris (black arrows) were retrieved by aspiration with minimal blood clots, consistent with fungal emboli (which was subsequently confirmed by culture).

\section{Discussion}

In this case, a significant aortic bioprosthetic valvular vegetation and paravavular abscess were present in the setting of persistent fungemia despite multiple weeks of intravenous anti-fungal therapy. Acute embolic occlusion of a large circumflex by fungal vegetation led to a STEMI and cardiac arrest. Our inhospital STEMI response team diagnosed the STEMI timely, and emergent primary PCI for reperfusion therapy with aspirational thrombectomy and balloon angioplasty resulted no-reflow phenomenon, likely due to large burden of distal fungal emboli. High fungal emboli burden were also represented by the history of systemic embolic complications in mesenteric and iliac arteries in this patient.

Fungal endocarditis is rare but carries a significant mortality risk. It is fatal in about $50 \%$ of the patients [1,2]. Patients with prosthetic valves are at increased risk of fungal endocarditis than those with native valves [3]. Fungal endocarditis is more prone to systemic embolization due to the larger size and friable nature of these vegetations [4,5]. Acute coronary syndromes (ACS) in the setting of infective endocarditis carry a high mortality rate, reported to be $64 \%$ in one of the studies [6]. The incidence of embolic ACS in this study was reported to be $0.51 \%$ [6]. Aortic valve vegetations are less commonly associated with embolic ACS due to the proximity to the coronary ostium [7]. The presence of a periaortic abscess should also be considered in patients with embolic ACS. The left anterior descending artery is most commonly involved in coronary embolization secondary to infective endocarditis due to its descending course.

The management of patients with STEMI due to septic emboli poses a significant clinical challenge. There are no clinical 
practice guidelines for these patients. Given the ECG evidence of potential transmural myocardial injury and infarction, these patients with ST-segment elevations are taken emergently to the cardiac catheterization laboratory, following the standard of care for patients with a traditional STEMI. Aspiration thrombectomy can be utilized in an attempt to remove the emboli; however, variable rates of success have been reported [8]. Revascularization with stenting should be avoided due to the risks of stent infection and formation of mycotic aneurysms. Another consideration is the intracoronary administration of thrombolytics in an attempt to break up the emboli, however, since they are primary infective and not thrombi, it appears to have limited to no clinical benefit. Systemic thrombolytic therapy has been used successfully in certain cases of septic coronary emboli but carries a high risk of intracranial hemorrhage and hemorrhagic conversion in patients with cerebrovascular manifestations of embolic phenomenon [9]. This case illustrates that septic coronary emboli can be a fatal complication of fungal bioprosthetic valvular endocarditis particularly when an early surgical strategy may not be effective or safe.

\section{References}

1. Pierrotti LC, Baddour LM (2002) Fungal endocarditis, 1995-2000. Chest 122(1): 302-310.
2. Badiee P, Amirghofran AA, Ghazi Nour M, Shafa M, Nemati MH (2014) Incidence and outcome of documented fungal endocarditis. Int Cardiovasc Res J 8(4): 152-155.

3. Boland JM, Chung HH, Robberts FJ, Wilson WR, Steckelberg JM, et al (2011) Fungal prosthetic valve endocarditis: Mayo Clinic experience with a clincopathologic analysis. Mycoses 54(4): 354-360.

4. Ellis ME, Al-Abdely H, Sandridge A, Greer W, Ventura W (2001) Fungal endocarditis: evidence in the world literature, 1965- 1995. Clin Infect Dis 32(1): 50-62.

5. Montague NT, Sugg WL (1974) Candida endocarditis with femora emboli. Treatment with surgery and 5-fluorocytosine. J Thorac and CardiolSurg 67(2): 322-327.

6. Manzano MC, Vilacosta I, San Roman JA, Aragoncillo P, Sarriá C, et al (2007) Acute coronary syndrome in infective endocarditis. Rev Esp Cardiol 60(1): 24-31.

7. Kessavane A, Marticho P, Zogheib E, Lorne E, Dupont H, et al. (2009) Septic coronary embolism in aortic valvular endocarditis. J Heart Valve Dis 18(5): $572-574$.

8. Maqsood K, Sarwar N, Eftekhari H, Lotfi A (2014) Septic coronary artery embolism treated with aspiration thrombectomy: case report and review of literature. Tex Heart Inst J 41(4): 437-439.

9. Hunter AJ, Girard DE (2001) Thrombolytics in infectious endocarditis associated myocardial infarction. J Emerg Med. 21(4): 401-406.

Your next submission with Juniper Publishers will reach you the below assets

- Quality Editorial service

- Swift Peer Review

- Reprints availability

- E-prints Service

- Manuscript Podcast for convenient understanding

- Global attainment for your research

- Manuscript accessibility in different formats ( Pdf, E-pub, Full Text, Audio)

- Unceasing customer service

Track the below URL for one-step submission https://juniperpublishers.com/online-submission.php 